\title{
Perianal Fistulizing Crohn's Disease: No Shortcuts to a Deep Understanding of the Disease
}

\author{
Jana G. Hashash ${ }^{1}$. Fadi H. Mourad ${ }^{1}$ \\ Published online: 24 August 2020 \\ (c) Springer Science+Business Media, LLC, part of Springer Nature 2020
}

Perianal fistulizing Crohn's disease (PFCD) is an aggressive and debilitating phenotype of Crohn's disease (CD) affecting up to one-third of $\mathrm{CD}$ patients. Its management requires a multidisciplinary approach involving inflammatory bowel disease (IBD) subspecialists, colorectal surgeons, and gastrointestinal radiologists. Despite advancements in the treatment of luminal CD, PFCD management remains challenging since remission rates do not exceed $50 \%$ with the use of combination medical and surgical approaches [1]. Many questions remain unanswered for PFCD patients including optimal therapeutic targets, prognostic factors for response, optimal biologic dose, target therapeutic drug levels, and determining the best timing in order to assess for fistula healing and the most accurate way to do so.

In this issue of Digestive Diseases and Sciences, Zhu et al. [2] published a retrospective study: "The effectiveness of infliximab on deep radiologic remission in Chinese patients with perianal fistulizing Crohn's disease." The authors investigated the effectiveness of infliximab on PFCD healing as documented during follow-up using clinical and magnetic resonance imaging (MRI) acquired data aimed at identifying predictors of achieving deep radiologic remission. Results demonstrated high perianal fistula healing rates with infliximab, a rate that increased with prolongation of treatment [2]. A higher trough level of infliximab and concomitant treatment with azathioprine increased fistula healing rates further. Prognostic factors for poor healing rates included younger age at diagnosis of $\mathrm{CD}$, proctitis, and the need for perianal surgery. The authors concluded that routine MRI should be used to monitor the healing of PFCD.

Patients with PFCD undergo testing to better characterize the anatomy of the fistula tract with either MRI or endoscopic ultrasound. An examination under anesthesia is

Jana G. Hashash

ja38@aub.edu.lb

1 Division of Gastroenterology, American University of Beirut, Beirut, Lebanon sometimes necessary though sigmoidoscopy is always performed in order to assess for the presence of endoscopic disease in the rectum.

Although not formally validated, there are several clinical outcome measures used to assess severity of PFCD, including the Anal Disease Activity Index, the Perianal Disease Activity Index (PDAI), the Fistula Drainage Assessment, and Pikarsky's Perianal Crohn's Disease Activity Index. The ultimate goal of therapy in PFCD is complete fistula closure, although in many patients, the best achievable outcome is symptomatic improvement. It is crucial to recognize that clinical remission does not reflect deep tissue healing; radiologic healing as assessed by MRI lags behind clinical remission by a median of 12 months [3]. Correlation of MRI (using the Van Assche score) with PDAI is weak, with no correlation with CRP [4].

Relying only on clinical remission in patients who have persistent inflammation as demonstrated with MRI leads to a high relapse rate after discontinuation of treatment [4]. Patients with perianal fistula treated with infliximab and who achieved deep healing as demonstrated with MRI had a significantly decreased rate of fistula recurrence compared with those who achieved only clinical remission [5]. Flarefree and perianal event-free survival were statistically longer in patients with deep radiologic remission compared with patients with abnormal MRI. Patients with active inflammation as demonstrated with MRI required four times more perianal surgeries compared with patients who were in deep radiographic remission. Therefore, MRI is an accurate noninvasive follow-up modality for PFCD. Achieving deep radiologic remission is a prognostic indicator for lower recurrence rates and should serve as a useful target in the management of these patients.

In luminal $\mathrm{CD}$, combination azathioprine and infliximab treatment was superior to monotherapy using either drug alone [6]. Zhu et al. [2] showed that concomitant treatment of azathioprine with infliximab increased the fistula healing rate compared with infliximab monotherapy (50\% vs 36.9\%, 
$P<0.001$ ), understandable given that PFCD is considered a severe form of $\mathrm{CD}$. Interpretation of these data is tempered by the observation that high-quality data do not exist in PFCD.

Infliximab is an effective treatment for fistula closure of PFCD [7, 8]. Initial studies recommended administration of $5 \mathrm{mg} / \mathrm{kg}$ of infliximab since a higher dose $(10 \mathrm{mg} / \mathrm{kg})$ was not associated with superior outcomes [7]. More recently, a number of studies showed that the median levels of circulating serum infliximab levels were higher in patients who clinically responded to infliximab as compared with those who did not respond ( $4.1 \mathrm{vs} 0.14 \mu \mathrm{g} / \mathrm{mL}, P=0.01$ ) [9]. This study showed that infliximab drug levels of $9.25 \mu \mathrm{g} / \mathrm{mL}$ at week 2 and $7.25 \mu \mathrm{g} / \mathrm{mL}$ at week 6 were the best predictors of fistula response by weeks 14 and 30 [9]. Another study of 117 patients with PFCD who were treated with infliximab for at least 24 weeks showed that patients with clinical fistula healing had higher serum infliximab levels compared with patients with active drainage ( $15.8 \mathrm{vs} 4.4 \mu \mathrm{g} / \mathrm{mL}, P=0.0001$ ) [10]. The authors concluded that for treating perianal fistulas, infliximab levels $>10 \mu \mathrm{g} / \mathrm{mL}$ might be needed to achieve the outcome of complete healing. These data strongly suggest that optimal treatment of PFCD with infliximab requires significantly higher target serum infliximab levels during both the induction and maintenance phases of treatment. Prospective studies are needed to determine the therapeutic infliximab level and optimal dose needed for patients with PFCD. Similarly, Zhu et al. demonstrated a concentrationdependent effect in patients who are in radiologic remission. Interestingly, however, the cutoff value for predicting radiologic remission of fistula was $2.9 \mu \mathrm{g} / \mathrm{mL}$, much lower than what is reported in non-Chinese individuals, likely related to the genetic background of Chinese patients and their susceptibility and response rates to infliximab [2]. The present study did not provide data on the effect of increasing the dose of infliximab on healing rates, which would have been interesting especially among the non-responders who had very low infliximab levels.

Early initiation of anti-tumor necrosis factor (anti-TNF) therapies has been associated with improved outcomes and prognosis in CD. Patients with PFCD who are considered to have severe $\mathrm{CD}$ and are at high risk for inferior outcomes may benefit from the early introduction of infliximab. Although this is the practice of many gastroenterologists, data on the effectiveness and outcomes of early initiation of anti-TNF for PFCD are still lacking.

Predictors of deep remission and response to therapy are important factors for patients and their treating physicians. Zhu et al. [2] showed that younger age at CD diagnosis, proctitis, and need for perianal fistula-related surgery were poor prognostic factors to healing using infliximab after 6 infliximab infusions. After 18 infliximab infusions, however, these factors lost significance. Other studies showed that active rectal inflammation (proctitis) and low BMI may be indicators of poor response to infliximab while having a simple fistula served as a good predictor of healing.

In conclusion, PFCD remains a burdensome phenotype of CD affecting a patient's physical and psychological wellbeing. A multidisciplinary approach with an IBD subspecialist, colorectal surgeon, and experienced gastrointestinal radiologist is required to achieve the best outcomes. Although infliximab remains the most studied and most successful biologic for the treatment of PFCD, its optimal dosing, therapeutic levels and the contribution of proactive drug monitoring to healing are yet to be determined. These questions should be clarified through prospective controlled studies. Though MRI is a noninvasive and reliable test used to monitor fistula healing, there are currently no clear established data regarding surveillance intervals and when to shift to another biologic, all management points that should be informed by further research.

\section{References}

1. Sandborn WJ, Fazio VW, Feagan BG, Hanauer SB. AGA technical review on perianal Crohn's disease. Gastroenterol. 2003;125:1508-1530.

2. Zhu M, Xu X, Feng Q, et al. The effectiveness of infliximab on deep radiological remission in Chinese Patients with perianal fistulizing Crohn's Disease. Dig Dis Sci. (Epub ahead of print). https ://doi.org/10.1007/s10620-020-06398-w.

3. Tozer P, Ng SC, Siddiqui MR, et al. Long-term MRI-guided combined anti-TNF- $\alpha$ and thiopurine therapy for Crohn's perianal fistulas. Inflamm Bowel Dis. 2012;18:1825-1834.

4. Horsthuis K, Ziech MLW, Bipat S. Evaluation of an MRI-based score of disease activity in perianal fistulizing Crohn's disease. Clin Imaging. 2011;35:360-365.

5. Chambaz M, Verdalle-Cazes M, Desprez C, et al. Deep remission on magnetic resonance imaging impacts outcomes of perianal fistulizing Crohn's disease. Dig Liver Dis. 2019;51:358-363.

6. Colombel JF, Sandborn WJ, Reinisch W. Infliximab, azathioprine, or combination therapy for Crohn's disease. $N$ Engl J Med. 2010;362:1383-1395.

7. Present DH, Rutgeerts $\mathrm{P}$, Targan S, et al. Infliximab for the treatment of fistulas in patients with Crohn's disease. $N$ Engl J Med. 1999;340:1398-1405.

8. Sands B, Van Deventer S, Bernstein C, et al. Long-term treatment of fistulizing Crohn's disease: response to infliximab in the ACCENT II trial through 54 weeks (abstr). Gastroenterology. 2002;122:A81.

9. Davidov Y, Ungar B, Bar-Yoseph H, et al. Association of induction infliximab levels with clinical response in perianal Crohn's disease. J Crohns Colitis. 2017;11:549-555.

10. Yarur AJ, Kanagala V, Stein DJ, et al. Higher infliximab trough levels are associated with perianal fistula healing in patients with Crohn's disease. Aliment Pharmacol Ther. 2017;45:933-940.

Publisher's Note Springer Nature remains neutral with regard to jurisdictional claims in published maps and institutional affiliations. 